\title{
Brain Computer Interface: Assessment of Spinal Cord Injury Patient towards Motor Movement through EEG application
}

\author{
Syahrull Hi-Fi Syam ${ }^{1}$, Heba Lakany², R.B. Ahmad ${ }^{3}$ and Bernard A. Conway² \\ ${ }^{1}$ Polytechnic of Tuanku Syed Sirajuddin, 02600, Perlis, Malaysia \\ ${ }^{2}$ Biomedical Engineering, University of Strathclyde G4 0NW, Glasgow, United Kingdom. \\ ${ }^{3}$ Faculty of Informatics and Computing, Universiti Sultan Zainal Abidin (UniSZA), Kuala Terengganu, Malaysia
}

\begin{abstract}
Electroencephalography (EEG) associated with motor task have been comprehensively investigated and it can also describe the brain activities while spinal cord injury (SCI) patient with para/tetraplegia performing movement with their limbs. This paper reviews on conducted research regarding application of brain computer interface (BCI) that offer alternative for neural impairments community such as spinal cord injury patient (SCI) which include the experimental design, signal analysis of EEG band signal and data processing methods. The findings claim that the EEG signals of SCI patients associated with movement tasks can be stimulated through mental and motor task. Other than that EEG signal component such as alpha and beta frequency bands indicate significance for analysing the brain activity of subjects with SCI during movements.
\end{abstract}

\section{Introduction}

Spinal cord injury (SCI) is an incapacitating disorder that affects physical and psychological health which lead to loss of motor and sensory function [1], increased risk of depression, on-going pain syndromes, profound lifestyle change, drug dependence as well as increased hospital admissions and decreased general health $[2,3]$. SCI may be caused either by trauma or disease and consequences of the SCI may drive the patient to be discriminated, isolated and restricted from normal daily life activity due to the motor and sensory disabilities.

Despite the limitation of independency, there are number of modalities which can offer the SCI population an assessment tool and a significance level of recovery through invasive or non-invasive method. For instance stem cells method, engineering approach and brain computer interface (BCI) approach. Among these 3 approaches BCI offer more applicable choice due to its reliability and promising results. This is because the stem cells method restrains by financial issue [4] and lack of independent validation's results of the various stem cell treatments [5]. Apart from that engineering approach induced modest behavioral which need further investigation.

BCI applies electroencephalography (EEG) signal to communicate with augmentative and assistive devices which offer an alternative to those who suffered from motor and sensory disabilities due to the SCI. BCI cover various type of application and one of it is derivation information from EEG signal regarding performing motor task and imaginary performing motor task [6]. In order to derive this information, it is essential to improve the EEG signal processing techniques and selection of features. By achieving this, the speed and accuracy of communication of the interface devices can be improved [7] and the training time of using it can be reduced.

\section{Experimental Design}

In recent years, there have been advances in a numbers of research investigating and developing successful BCI endeavors to improve the speed and accuracy of communication of these interfaces. In order to achieve this goal, multidimensional approaches and modalities have been implemented involving mental task [6], [8], [9] and various motor tasks utilising finger [10]-[14], hand [15]-[17],[19], foot [16], [18], [19], and tongue movement [19].

Craig and Nguyen [8] design four different mental tasks for patients with chronic SCI. The mental tasks which were chosen to evoke changes in brain activity were intended to generally give results which tended to favor one hemisphere over the other, as the intention of the research is to use the differences in EEG over the two hemispheres to help with the classification. The results established that the 'stop-start' classifier has achieved a total accuracy of $94 \%$, while the 'left-right' classifier has achieved approximately $77.5 \%$ accuracy.

Studies carried by Erbil \& Ungan [14], investigate whether the suppression that is known to occur during the onset of a single movement would persist or otherwise habituate when the movement is continuously repeated for a long period of time. They designed experimental protocol which required subjects to perform repetitive simultaneous extension-flexion of the fingers II-V in one hand, continuously for a period of at least $30 \mathrm{~s}$ with interval of rest of $30 \mathrm{~s}$. The finding demonstrated that the sustained and continuous movements are conducted through quite different processes and modulation of the beta amplitude, rather than the changes in alpha amplitude, appeared to be more closely correlated with the execution of a continuous movement.

Apart from that Boi et al [17], conduct a research exploring the effectiveness combinations of computational methods for prediction of movement intention preceding the production of self-paced right and left hand movements. They designed a protocol 
which require subjects to perform self-paced movements consisting of three key strokes with either hand. The results indicated that the discrimination accuracy was as high as $75 \%$ by utilizing one of the better combinations of independent component analysis (ICA), power spectral density estimation (PSD) and support vector machine (SVM). Results also claimed that beta band EEG activity of the channels over right sensorimotor cortex was most appropriate for discrimination of right and left hand movement intention.

On the other hand, Morash et al [18], investigating on neural signals preceding 4 movement (right hand, left hand, tongue, and right foot movements) and motor imagery in order to predict the occurrence of the four movements/motor imageries. They adapt an experimental protocol that require subjects to perform or kinaesthetically imagine four movements which instructed with a specific stimulus and performed at a second stimulus. The obtained results indicate that accuracy of movement/imagery preparation classification varied between subjects. It also include that high accuracies were associated with classifications relied heavily on the ERD/ERS in order to discriminate movement/imagery planning.

\section{Signal Analysis of EEG Band Signal}

EEG or brainwaves are electrical signals produced by excitatory and inhibitory potentials of neurons (electrical activity of an alternating type) recorded from the scalp surface after being picked up by metal electrodes and conductive media [20].

Normally EEG activity can be characterised as being within five distinct frequency bands, for instance delta $/ \delta$ $(0.1-3 \mathrm{~Hz})$, theta/ $\theta(4-7 \mathrm{~Hz})$, alpha/ $\alpha(8-13 \mathrm{~Hz})$, beta/ $\beta(14-29 \mathrm{~Hz})$ and Gamma/s $(30-40 \mathrm{~Hz})$ [21]. Numbers of study on BCI have been using one or a combination of these frequency bands in order to access variety of brain signal component such as visual evoked potentials [22], [23], slow cortical potentials [24], [25], P300 evoked potentials [26], [27], mu and beta rhythms [28],[29] and cortical neuronal action potentials [30].

Antonio Castro et al [31], extract the movementrelated brain potentials (MRBPs) consisting of the readiness potential, pre-motion positivity, motor potential and reafferent potential in order to reflect brain electrical activity associated with processes of movement preparation and movement execution.

On the hand, Boord et al [32], compared brain wave activity (frontal, central, parietal, occipital and temporal regions) between the able-bodied and SCI subjects utilizing the $8-13 \mathrm{~Hz}$ magnitude, peak amplitude and frequency of $8-13 \mathrm{~Hz}$ levels. Reporting in his other work [33] regarding comparing the peak frequency ( $\theta-\alpha$ band) and power ( $\delta, \theta, \alpha$ and $\beta$ bands) between able-bodied, no-pain, and pain groups; peak $\theta-\alpha$ band frequency (PTAF) was calculated as the frequency of peak power between 4 and $13 \mathrm{~Hz}$.

Apart from that Yong woong et al [34], examining $\mathrm{mu}$ and beta event-related desynchronization/ synchronization (ERD/ERS) during motor imagery tasks with varying movement duration and the potential impacts of movement duration on ERD/ERS patterns. This study measured ERD/ERS through mu $(8-13 \mathrm{~Hz})$ and beta bands $(14-30 \mathrm{~Hz})$. The ERD/ERS was quantified following standard calculation of Pfurtscheller and Aranibar [35].

According to Jean-Michel Guerit's article [36], on the main principles and indications of EEG and evoked potential (EP) neuromonitoring in the operating room; which claim that neuromonitoring in the operation room can assess the depth of anesthesia and prevent ischemic brain or spinal cord damage, mechanical injuries of the brain. At the same time it also may help anesthesiologist to control the level of sedation and aid surgeons to prevent neurological complication when they are performing an operation.

In Heba and Conway [37] studies, motion related potentials (MRP's) were recorded from the motor cortex area in the left hemisphere of brain through channel C3. MRP's were analyzed from $\alpha(8-12 \mathrm{~Hz}), \beta(18-24$ $\mathrm{Hz})$ and $в(28-37 \mathrm{~Hz})$ bands and the results from this study indicate that the different direction of recorded EEG signals of wrist movement can be discriminated using Euclidean distance classifier.

\section{Data Processing Methods}

Recorded EEG data are always being contaminated by artifacts, interfering noises due to the electronic amplifier, power line interference and external interference plus the skull which acts as a spatial lowpass band filter that eliminates much of the information generated intracortically [38]. Thus in order to build BCI that apply real time EEG signal as medium to communicate/ control augmentative, it is essential to improve the EEG signal processing techniques (preprocessing, features extracting and features classifying).

Pre-processing procedure is carried out as for removing the unwanted signal and noise by using spatiospectro-temporal filters. These filters can be simple frequency filters (low pass filter, band pass filter), independent component analysis (ICA) and common spatial pattern. For instance Heba and Conway [37] used low pass filter at $50 \mathrm{~Hz}$ for removing eye blink and movement artifacts. As for same purpose for preprocessing process, Yongwoong et al [34] also have implemented band pass filtered at $8-30 \mathrm{~Hz}$ and Boord et al [32] adopt ICA for removing eye blink and saccade artifacts.

Features extraction involves simplifying the amount of resources required to describe a large set of data accurately. In BCI endeavors, feature extraction taxonomy can be grouped broadly as time domain, frequency domain and time-frequency domain. Heba and Conway [37] use continuous wavelet transform (CWT) for extracting spatio-temporal characteristic of EEG during movement transition and movement planning. Navarro 'et al [38] extract features mainly on time based feature which include $50 \mathrm{~ms}$ window averages of rectified EEG amplitude, dominant frequency, timedomain variance over $2 \mathrm{~s}$ response and total power over $2 \mathrm{~s}$ response (using Welch's method) in order to classify 
wrist movement (flexion, extension, pronation, supination). In the other study by Antonio Castro [40] which focus in analysing the relationships between movement preparation and movement execution, the amplitude of each one of the MRBPs were measured as the dependent variable.

Features classifying is a process to assign automatically a class (mental task performed by BCI user) to the feature vector which previously extracted using algorithm known as classifiers. In BCI field most of the classifiers belongs to linear classifier, neural network, nearest neighbour and classifier combination. Craig and Nguyen [8] have implemented neural network classifier and suggested the inclusion of adaptive training of the classifier. In their work the classifier was able to generalize the result from subjects (5) and learn to recognize them with an overall success rate of $82.4 \%$. In the other study by Heba and Conway [38], classification of the extracted features vector was done by measuring Euclidean distance between the test sample and the representation vector. The classification's results are promising and prove that the suitability of the technique for the application.

\section{Conclusion}

This paper specifies the major role played by EEG as essential medium in BCI endeavors which offer alternative way of communicating and interacting with outside environment among the impairments community population. The promising and convincing results from the recent research demonstrate that, in the future BCI will be the main option for non-invasive method for controlling neuroprosthesis in neural impairments community such as SCI. Future works will focus more on investigating and discovering the effective modalities which can stimulate the organization of brain functional networks activated in presence of motor impairment and enhance the processing techniques on manipulating neural activity which can be volitionally modulated to sub serve training control strategy.

\section{References}

1. Krishnan R. V., Mythuswamy R., \& Sankar V., "Spinal cord injury repair research: A new combination treatment strategy," International Journal of Neuroscience, 108: 201-207, 2003.

2. Craig A., Hancock K., Dickson H., \& Chang E., "Immunizing against depression and anxiety following spinal cord injury," Archives of Physical Medicine and Rehabilitation, 79: 375-377, 1998.

3. Widerstrom-Noga E., Felipe-Cuervo E., \& Yezierski R., "Relationships among clinical characteristics of chronic pain after spinal cord injury," Archives of Physical \& Mental Rehabilitation, 82: 1191-1197, 2001.

4. Simon Frantz., "Embryonic stem cell pioneer Geron exits field, cut losses," Nature Biotechnology, 30: 12-13, 2012.
5. Michael G., \& Reaz Vawda., "Cellular treatments for spinal cord injury: The time is right for clinical trials," Neurotherapuetics, 8: 704-720, 2011.

6. Lakany H., \& Conway B.A., "Comparing EEG patterns of actual and imaginary wrist movements a machine learning approach," Proceedings of the first ICGST International Conference on Artificial Intelligence and Machine Learning AIML, ICGST, Cairo, Egypt, 05(5): 124-127, 2005.

7. Vaughan, T.M., "Brain-computer interface technology: a review of the second international meeting," IEEE Transactions on Neural Systems and Rehabilitation Engineering, 11:94-109, 2003.

8. Craig. D.A., Nguyen. H.T., "Adaptive EEG Thought Pattern Classifier for Advanced Wheelchair Control," Engineering in Medicine and Biology Society, 29th Annual International Conference of the IEEE: 2544 - 2547, 2007.

9. J.A. Pineda., B.Z Allison., and A. Vankov., "The Effects of Self-Movement, Observation and Imagination on $\mu$ Rhythms and Readiness Potentials (RP's): Towards a Brain-Computer Interface (BCI), IEEE Transactions on Rehabilitation Engineering, 8:219-222, 2000.

10. Stancák, A., Pfurtscheller, G., "Desynchronization and recovery of $\mathrm{b}$ rhythms during brisk and slow self-paced finger movements in man," Neuroscience Letters, 196: 21-24, 1995.

11. Cassim, F., Szurhaj, W., Sediri, H., Devos, D., Bourriez, J.L., Poirot, I., Derambure, P., Defebvre, L., Guieu, J.D., "Brief and sustained movements: differences in event-related (de)synchronization (ERD/ERS) patterns," Clinical Neurophysiology, 111: 2032-2039, 2000.

12. Haeger-Ross, C., Schieber, M.H., "Quantifying the independence of human finger movements: comparisons of digits, hands and movement frequencies," Journal of Neuroscience, 20(22):8542-8550, 2000.

13. Li, Y., Gao, X., Liu, H., Gao, S., "Classification of single-trial electroencephalogram during finger movement," IEEE Transactions on Biomedical Engineering, 51(6): 1019-1025, 2004.

14. Erbil, N., Ungan, P., "Changes in the alpha and beta amplitudes of the central EEG during the onset, continuation, and offset of long-duration repetitive hand movements," Brain Research, 1169: 44-56, 2007.

15. Pfurtscheller, G., Neuper, C., Pichler-Zalaudek, K., Edlinger, G., Lopes da Silva, F., "Do brain oscillations of different frequencies indicate interaction between cortical areas in humans?," Neuroscience Letters, 286:66-68, 2000.

16. Neuper, C., Pfurtscheller, G., "Evidence for distinct beta resonance frequencies in human EEG related to specific sensorimotor cortical areas," Clinical Neurophysiology, 112:2084-2097, 2001.

17. Bai, O., Lin, P., Vorbach, S., Li, J., Furlani, S., Hallett, M., "Exploration of computational methods for classification of movement intention during human voluntary movement from single trial 
EEG," Clinical Neurophysiology, 118:2637-2655, 2007.

18. Müller-Putz, G.R., Zimmermann, D., Graimann, B., Nestinger, K., Korisek, G., Pfurtscheller, G., "Event-related beta EEG-changes during passive and attempted foot movements in paraplegic patients," Brain Research, 1137:84-91, 2007.

19. Morash, V., Bai, O., Furlani, S., Lin, P., Hallett, M., "Classifying EEG signals preceding right hand, left hand, tongue, and right foot movements and motor imageries," Clinical Neurophysiology, 119:2570-2578, 2008.

20. Niedermeyer E, F. H. Lopes da Silva., "Electroencephalography: Basic principles, clinical applications and related fields,". 3rd edition, Lippincott, Williams \& Wilkins, Philadelphia, 1993.

21. Guyton. A.C. Textbook of Medical Physiology, Sixth Edition, W.B.Saunders Co., Philadelphia, Pa.6: 675-680, 1981.

22. Sutter, E.E., Tran, D., "The brain response interface: communication through visually induced electrical brain responses," Journal of Microcomputer Applications 15:31-45, 1992.

23. Middendorf, M., McMillan, G., Calhoun, G., Jones, K.S., "Braine computer interfaces based on the steady-state visual-evoked response," IEEE Transaction on Rehabilitation Engineering 8 (2):211-214, 2000.

24. Rockstroh, B., Elbert, T., Canavan, A., Lutzenberger, W., Birbaumer, N., "Slow Cortical Potentials and Behavior," second ed.. Urban and Schwarzenberg, Baltimore, MD, 1989.

25. Birbaumer, N., "Slow cortical potentials: their origin, meaning, and clinical use," In: van Boxtel, G.J.M., Bo€cker, K.B.E. (Eds.), Brain and Behavior Past, Present, and Future, Tilburg University Press, Tilburg, pp. 25-39, 1997.

26. Donchin, E., Spencer, K.M., Wijesinghe, R., "The mental prosthesis: assessing the speed of a P300based brain computer interface," IEEE Transaction on Rehabilitation Engineering 8:174-179, 2000.

27. Li, Y., Nam, C.S., Shadden, B., Johnson, S., "A P300-based Brain-Computer Interface (BCI): Effects of Interface Type and Screen Size," International Journal of Human-Computer Interaction 27 (1):52-68, 2011.

28. Neuper, C., Müller-Putz, G.R., Scherer, R., Pfurtscheller, G., "Motor imagery and EEG-based control of spelling devices and neuroprostheses," Progress in Brain Research 159:393-409, 2006.

29. Neuper, C., Scherer, R., Wriessnegger, S., Pfurtscheller, G., "Motor imagery and action observation: modulation of sensorimotor brain rhythms during mental control of a brain-computer interface," Clinical Neurophysiology 120:239-247, 2009.

30. Kennedy, P.R., Bakey, R.A.E., Moore, M.M., Adams, K., "Direct control of a computer from the human central nervous system," IEEE Transaction on Rehabilitation Engineering 8:198-202, 2000.
31. Antonio Castro, Fernando Diaz and Geert J. M. van Boxtel, "How does a short history of spinal cord injury affect movement-related brain potentials?," European Journal of Neuroscience, 25:2927-2934, 2007.

32. P Boord, Y Tran, J Middleton, A Craig, "Levels of brain wave activity $(8-13 \mathrm{~Hz})$ in persons with spinal cord injury," Spinal Cord. Houndsmills, 42(2): 73, 2004.

33. P Boord, P J Siddall, Y Tran, D Herbert, et al, "Electroencephalographic slowing and reduced reactivity in neuropathic pain following spinal cord injury," Spinal Cord. Houndsmills, 46(2): 118-124, 2008.

34. Yongwoong Jeon, Chang S. Nam, Young-Joo Kim, Min Cheol Whang, "Event-related (De) synchronization (ERD/ERS) during motor imagery tasks: Implications for brainecomputer interfaces," International Journal of Industrial Ergonomics, 41:428-436, 2011.

35. Pfurtscheller, G., Aranibar, A., "Evaluation of event-related desynchronization (ERD) preceding and following voluntary self-paced movement," Electroencephalography and Clinical Neurophysiology 46:138-146, 1979.

36. Jean-Michel Guérit, "Neuromonitoring in the operating room: why, when, and how to monitor?," Electroencephalography and Clinical Neurophysiology, 106(1): 1-21, 1998.

37. Heba Lakany and B.A. Conway, "Classification of Wrist Movements using EEG-based Wavelets Features," Proceedings of IEEE Engineering in Medicine and Biology, 27:5405-5407, 2005.

38. I. Navarro, F. Sepulveda, B. Hubais, "A Comparison of Time, Frequency and ICA Based Features and Five Classifiers for Wrist Movement Classification in EEG Signals,"Proceedings of the 2005 IEEE Engineering in Medicine and Biology,2118-2121, 2005.

39. Guger,W.H., Ramoser, H. \& Pfurtscheller,G., "Real-time EEG analysis with subject-spesific spatial pattern for brain computer interface," IEEE Transaction on Rehabilitation Engineering, 8(4), 2000.

40. Antonio Castro, Fernando Diaz and Geert J. M. van Boxtel, "How does a short history of spinal cord injury affect movement-related brain potentials?," European Journal of Neuroscience, 25: 2927-2934, 2007. 